\title{
Morfología y taxonomía de Scinaia halliae (Scinaiaceae, Rhodophyta) en el litoral de Bahia y Espírito Santo, Brasil
}

\author{
Morphological and taxonomic studies in Scinaia halliae (Scinaiaceae, Rhodophyta) on the coast of \\ Bahia and Espírito Santo, Brazil \\ José Marcos de C. Nunes ${ }^{1}$ y Silvia Maria P. B. Guimarães ${ }^{2}$ \\ ${ }^{1}$ Laboratório de Algas Marinhas - LAMAR, Departamento de Botânica, Instituto de Biologia, Campus de Ondina, \\ Universidade Federal da Bahia - UFBA, CEP 40170-280, Salvador, Bahia, Brasil \\ ${ }^{2}$ Instituto de Botânica, Seção de Ficologia, Av. Miguel Estéfano, 3687, \\ CEP 04301-902, São Paulo, SP, Brasil \\ jmcnunes@ufba.br
}

\begin{abstract}
Scinaia halliae (Scinaiaceae, Rhodophyta) is analysed morphologically and taxonomically from the coast of Espírito Santo and Bahia, Brazil. Specimens were collected on the intertidal and in infralitoral zones from $16 \mathrm{~m}$ depth through a dredge Holme. Descriptions and illustrations are presented, as well as comments on habitat and geographical distribution
\end{abstract}

in the Brazilian coast. This study confirms that Scinaia brasiliensis Joly \& Cordeiro-Marino is synonym of $S$. halliae (Setchell) Huismann, and this species is recorded, for the first time, from the coast of Bahia.

Key words: Seaweeds, Nemaliales, diversity, Scinaia brasiliensis

\section{Introducción}

La familia Scinaiaceae fue separada de la familia Galaxauraceae por Huisman et al. (2004) basándose en estudios moleculares. En ese estudio se reconocieron tres familias del orden Nemaliales: Liagoraceae, que comprende géneros previamente ubicados en dos familias, Nemaliaceae y Dermonemataceae; Galaxauraceae, que incluye los géneros Actinotrichia Decaisne, Galaxaura Lamouroux (sensu lato) y Tricleocarpa Huisman \& Borowitzka; y la nueva familia Scinaiaceae, que comprende los géneros Scinaia Bivona, Gloiophloea Agardh, Nothogenia Montagne y Whidbeyella Setchell \& Gardner (Huisman et al. 2004). Los cuatro géneros de Scinaiaceae se caracterizan por presentar talo sin calcificación, córtex consolidado, pericarpo distinto, espermatangios originados en la superficie del talo formando soros (Scinaia y Gloiophloea) o en pequeñas cavidades (Nothogenia) y un ciclo de vida marcadamente heteromórfico con tetrasporófito costroso o filamentoso. El mismo estudio indica que Scinaiaceae es un grupo que divergió recientemente dentro de Nemaliales y es considerado un grupo hermano de Liagoraceae. Recientemente, Lee \& Ko (2006) realizaron estudios en las especies Scinaia de Corea. León-Cisneros et al. (2009) hicieron una nueva evaluación del género Scinaia del Golfo de California sobre la base de las colecciones históricas y recientes.
Actualmente, el género Scinaia comprende 43 especies aceptadas (Guiry 2009). Huisman (1985) examinó detalladamente las especies-tipo de los géneros Scinaia, Gloiophloea, Pseudoscinaia Setchell y Pseudogloiophloea Levring, concluyendo que estos dos últimos no presentaban características suficientes para justificar su reconocimiento. Este autor aceptó como válidos el género Scinaia, que se caracteriza por presentar córtex con células infladas incoloras (utrículos) y el género Gloiophloea, con córtex constituido por células pigmentadas y utrículos ausentes.

Las especies de Scinaia referidas para el Atlántico tropical americano son: Scinaia halliae (Setchell) Huisman (Venezuela y Florida: Ganesan 1974, Taylor 1960; Colombia: Schnetter \& Bula-Meyer 1978); S. caribaea (Taylor) Huisman (Haití: Taylor 1943; Venezuela: Ganesan 1990; Hispaniola: BetancourtFernández \& Herrera-Moreno 2001); S. complanata (Collins) Cotton (Golfo de México y Caribe: Ortega et al. 2001; Venezuela: Ganesan 1990); S. complanata (Collins) Cotton var. intermedia Børgesen (México: Robledo et al. 2003; Florida: Hanisack \& Blair 1988; Colombia: Díaz-Pulido \& Díaz-Ruíz 2003); S. furcellata (Turner) J. Agardh (Venezuela: Ganesan 1990; Golfo de México y Caribe: Ortega et al. 2001); y S. incrassata Eiseman (Florida: Hanisack \& Blair 1988; Colombia: Díaz-Pulido \& Díaz-Ruíz 2003). 
Las siguientes especies de Scinaia han sido citadas para Brasil: S. brasiliensis (Joly \& Cordeiro-Marino) Huisman (Joly et al. 1965, como Pseudogloiophloea brasiliensis Joly \& Cordeiro-Marino), S. complanata (Collins) Cotton (Joly et al. 1966) y S. furcellata (Turner) J. Agardh (Joly et al. 1965). Recientemente, Guimarães (2006) citó para el Estado de Espírito Santo las especies Scinaia cf. aborealis Huisman, S. complanata y S. halliae (Setchell) Huisman.

Este estudio tiene como objetivo describir los ejemplares de Scinaia halliae (Setchell) Huisman encontrados en el litoral de Bahia y Espírito Santo, presentando iconografías detalladas y diversas consideraciones sobre la propuesta de sinonimización de las especies Scinaia brasiliensis y S. halliae (Guimarães 2006). El presente estudio integra los Proyectos: 'Algas marinhas bentônicas do litoral da Bahia, Brasil’ desarrollado por la Universidad Federal de Bahía asociada con la Universidad del Estado de Bahía, y 'Rodofíceas Marinhas Bentônicas do Estado do Espírito Santo’ desarrollado por el Instituto de Botánica, São Paulo.

\section{Material y métodos}

Las muestras fueron recogidas en el litoral de Bahía $\left(11^{\circ} 27^{\prime} \mathrm{S}-37^{\circ} 20^{\prime} \mathrm{W}\right.$ y $\left.18^{\circ} 20^{\prime} \mathrm{S}-39^{\circ} 39^{\prime} \mathrm{W}\right)$ en puntos de la Bahía de Todos los Santos (BTS) y en los municipios de Cairú, Camaçari y Salvador. Los muestreos fueron realizados en el infralitoral mediante el uso de draga tipo Holme hasta los $16 \mathrm{~m}$ de profundidad. Los ejemplares fueron recogidos fijados a rodolitos de algas calcáreas incrustrantes. En el Estado do Espírito Santo (18²0'S $39^{\circ} 40^{\prime} \mathrm{W}$ y $21^{\circ} 18^{\prime} \mathrm{S}-40^{\circ} 57^{\prime} \mathrm{W}$ ), los ejemplares fueron recogidos en localidades al norte y al sur de Vitória, creciendo fijos a las rocas en la zona intermareal.

El material recolectado se fijó con formalina al 4\%, para su posterior estudio anatómico. Los ejemplares depositados en los herbarios ${ }^{1}$ SPF, SP, HUNEB y ALCB también fueron analizados en este estudio.

Los especímenes fueron observados detalladamente bajo microscopio óptico, mediante la preparación de portaobjetos semi-permanentes. También se hicieron cortes transversales a mano alzada con cuchilla de afeitar, teñidos con azul de anilina a $1 \%$, acidificada con $\mathrm{HCl}$ $1 \mathrm{~N}$. Se hicieron registros fotográficos utilizando un fotomicroscopio con equipo de captura de imagen (cámara Sony ${ }^{\circledR}$ acoplada a microscopio Zeiss ${ }^{\circledR}$ ).

${ }^{1}$ Herbário da Universidade de São Paulo (SPF); Herbário Maria Eneyda P.K. Fidalgo (SP); Herbário da Universidade do Estado da Bahia (HUNEB) y Herbário Alexandre Leal Costa (ALCB).
El material fue identificado, descrito y iconografiado, para posteriormente proceder a la preparación de los pliegos de herbario para incluirlos en el Herbário Alexandre Leal Costa (ALCB) de la Universidad Federal de Bahia y en el Herbário Maria Eneyda P.K. Fidalgo (SP) del Instituto de Botánica de São Paulo.

\section{Resultados y discusión}

\section{Scinaia halliae (Setchell) Huisman}

Sinonímias: Scinaia brasiliensis (Joly \& CordeiroMarino) Huisman; Gloiophloea halliae Setchell.

Talo cilíndrico, coloración rósea-rojiza a vinácea, consistencia blanda, erecto a partir del disco de fijación. Frondas con hasta $30 \mathrm{~cm}$ de longitud, ramificación dicotómica (Fig. 1a), 9-13 dicotomías en ángulo agudo distanciadas por 0,8-3,0 cm, suave constricción en la base de las dicotomías. Ápices redondeados (Fig. 1b). Estipe cilíndrico, poco diferenciado, 1,0-1,5 cm de longitud. Diámetro de los segmentos por encima de las dicotomías: en la región apical 1-2 mm, en la región mediana 2-3 mm y en la región basal 1-2 mm. Cutícula visible. En vista superficial, capa cortical con células grandes incoloras (utrículos) rodeadas de utrículos más pequeños y por células pigmentadas (Fig. 1c). En la región mediana del talo, capa cortical superficial con utrículos yuxtapuestos, con 15-38 $\mu \mathrm{m}$ de longitud y 10-23 $\mu \mathrm{m}$ de anchura. Abajo de la capa de utrículos, células hipodérmicas pigmentadas, redondeadas con 7-10 $\mu \mathrm{m}$ de diámetro, dispuestas en 1-2 capas. Ocasionalmente células hipodérmicas penetrando entre utrículos y atingiendo la superficie de la córtex. Médula con eje central de filamentos con 3-15 $\mu \mathrm{m}$ de diámetro, de éstos, se irradian otros filamentos con 2-3 $\mu \mathrm{m}$ de diámetro, conectándose a las células hipodérmicas. Ejemplares monoicos. Soros masculinos en manchas irregulares (Fig. 1d, e), entre los cistocarpos, en las regiones superiores y apicales del talo. Cistocarpos en toda la fronda, con excepción de la región basal, con 140-180 $\mu$ m de diámetro, inmersos, abriéndose por un poro. Ramo carpogonial con tres células, carpogonio cónico y tricógino corto. Debajo del carpogonio, la célula hipógina (Fig. 1f) separa por divisiones laterales oblicuas dos células hijas, una de cada lado; una de estas células se divide nuevamente, formando una tercera célula hija. La célula hipógina y las tres células hijas se disponen de manera cruzada. La célula basal del ramo carpogonial presenta filamentos estériles los cuales forman parte del pericarpo. Origen del gonimoblasto a partir del carpogonio. Cistocarpo maduro con pericarpo compuesto de numerosos filamentos. Célula de fusión compuesta por la célula hipógina fusionada con el carpogonio (Fig. 1g, h). Las ramas espermatangiales 


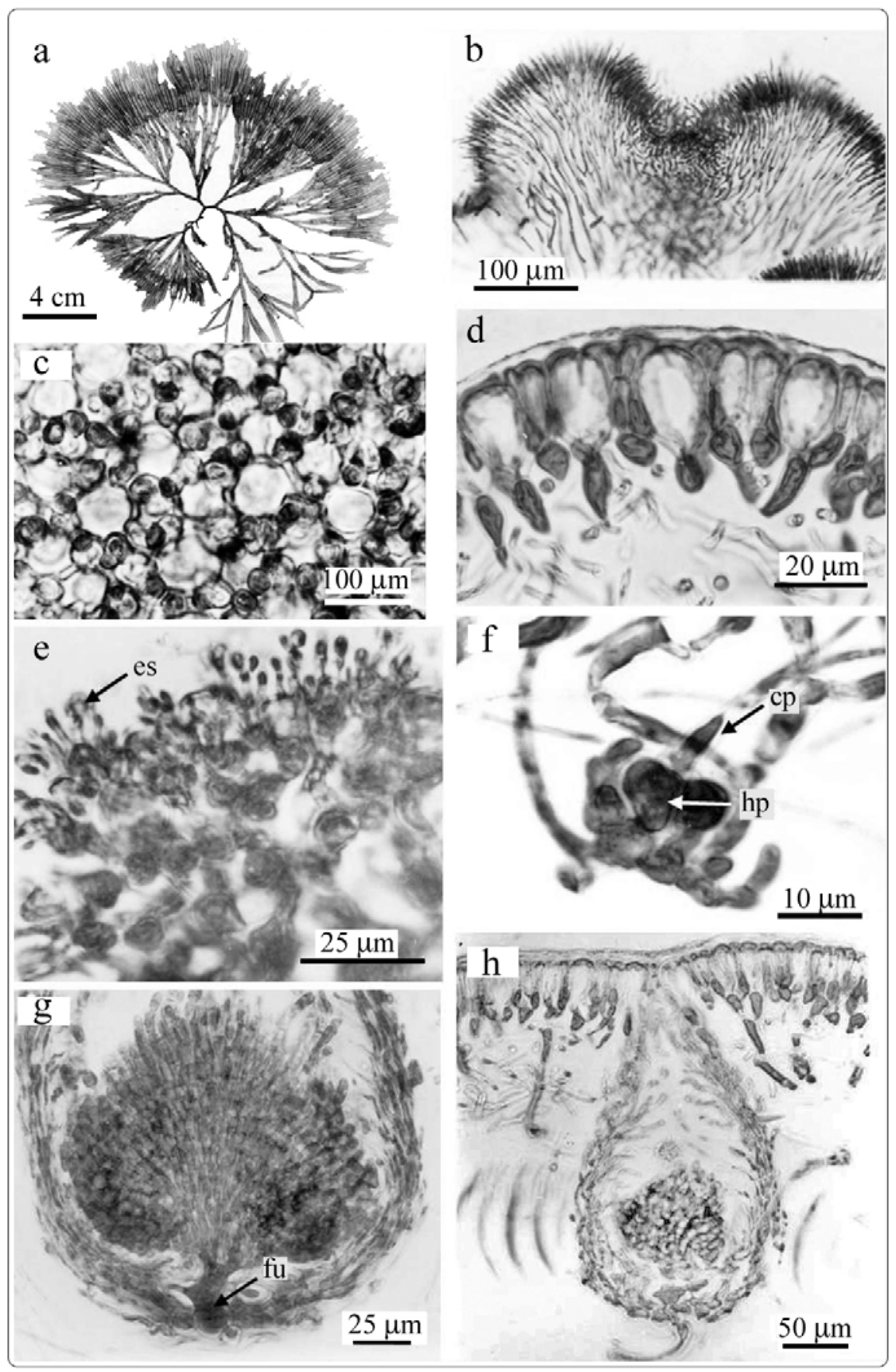

Figura 1

Scinaia halliae: a) hábito; b) corte longitudinal del talo en el ápice; c) vista superficial de las células corticales de tamaños distintos; d) corte transversal enseñando la región cortical; e) vista superficial de los soros espermatangiales con espermatangios (es); f) rama carpogonial con célula hipógina (hp) y carpogonio (cp); g) corte transversal del cistocarpo enseñando la célula de fusión (fu); h) corte transversal del talo enseñando cistocarpo maduro

Scinaia halliae: a) habit; b) longitudinal section of thallus in the apex region; c) superficial view of the cortical cells of different sizes; d) cross section showing the cortical cells; e) superficial view of the spermatangia sorus (es); f) branch carpogonial with hypoginous cell (hp) and carpogonium (cp); g) cross section showing the mature cystocarp and cell fusion (fu); h) cross section of thallus showing the mature cystocarp 
surgen como dos o tres células alargadas que se forman a partir de las células hipodérmicas y penetran a través de los utrículos. Estas células alargadas producen dos a tres células madres de espermatangios, en cuyos ápices se diferencian de 1 a 3 espermatangios.

Material examinado: BRASIL, ALAGOAS, Maceió, Playa do Pimenta, E.C. Oliveira, 31.01.1965 (SPF 2016 - isotipo (como Scinaia brasiliensis). BAHIA, Baía de Todos los Santos, $13^{\circ} 23^{\prime} 00^{\prime \prime S}$; $38^{\circ} 88^{\prime} 00^{\prime \prime} W$, Dutra, L.X, 27.07.03, (ALCB 65149); Cairú, 13²5’23,9"S; 38 50'25.9' W, ENSR, 07.01.04, (ALCB 64966); Camaçari, Arembepe, Cepemar, 12.03.94, (ALCB 22157); Jauá, Nunes, J.M. de C., 23.03.85, (ALCB 17214); Salvador, Pituba, Boccanera, N.B., 24.01.89 (ALCB 32278); Nunes, J.M. de C., 17.04.07 (HUNEB 11304); Stella Maris, Nunes, J.M. de C., 13.01.03, (ALCB 57589); 01.01.07 (HUNEB 10190). ESPÍRITO SANTO, Aracruz, Barra do Riacho, Guimarães, S.M.P.B. 13.10.1985 (SP 400062); Playa dos Padres, Guimarães, S.M.P.B., 14.06.1995 (SP 400063); Itapemirim, costa entre Itaoca y Itaipava, Guimarães, S.M.P.B., 18.04.1988 (SP 400064); Guimarães, S.M.P.B., 12.11.1989 (SP 400065).

Distribución en el litoral brasileño: Alagoas (como Scinaia brasiliensis, Joly et al. 1965), Bahia (presente estudio) y Espírito Santo (Guimarães 2006; presente estudio).

Huisman (1986) consideró dos grupos de especies de Scinaia, los cuales difieren en cuanto a la disposición de las células corticales: el grupo de $S$. furcellata, cuyas especies presentan el córtex compuesto de utrículos inflados y de células más pequeñas, frecuentemente pigmentadas (ej. S. furcellata, S. halliae, S. brasiliensis, S. caribaea, S. australis (Setchell) Huisman, S. proliferata Huisman) y el grupo de S. moniliformis cuyas especies presentan el córtex compuesto casi totalmente, de utrículos de tamaño aproximadamente uniforme (ej. S. moniliformis Agardh, S. howensis Huisman, S. aborealis Huisman, $S$. tsingalensis Tseng, S. huismanii Vroom \& Abbott).

Los ejemplares estudiados pertenecen al grupo de Scinaia furcellata. S. furcellata, S. halliae y $S$. brasiliensis, son especies próximas al material estudiado en este trabajo, por tener hábito cilíndrico, prácticamente sin constricciones.

En la comparación de las características de estas tres especies, basándose en Setchell (1914), Taylor (1960), Joly et al. (1965), Huisman (1985) y Ganesan (1974), se verificó que la morfología interna, las dimensiones de los utrículos y de las células hipodérmicas son características muy próximas y, por lo tanto, no ofrecen criterios adicionales para su separación.

Scinaia furcellata fue estudiada por Huisman (1985) quién examinó además el material tipo, presentando estudios detallados de la morfología vegetativa y reproductiva. Los datos de Huisman (1985) fueron comparados con los obtenidos en este trabajo, verificándose que los ejemplares de Alagoas, Bahia y Espírito Santo son muy semejantes a $S$. furcellata, difiriendo básicamente por la longitud, pues los especímenes estudiados alcanzan $30 \mathrm{~cm}$. En cuanto $S$. furcellata (localidad tipo: Sheringham, Norfolk, England) tiene como máximo $10 \mathrm{~cm}$ (Dixon \& Irvine 1977, Huisman 1985, Setchell, 1914). Las relaciones entre Scinaia halliae y $S$. furcellata no están claras; entretanto, Huisman (1985) consideró S. furcellata como endémica del noroeste de Europa, Mar Mediterráneo y norte de África.

Las dos especies brasileñas identificadas como Scinaia complanata y $S$. furcellata fueron descritas por Joly (1965) para el litoral del Estado de São Paulo. Aún cuando han sido encontradas posteriormente en otros estudios, estas especies no han tenido una descripción completa, sin ilustraciones respectivas. Los caracteres del talo en cuanto a monoica o dioica, patrón de distribución de los utrículos, número de ramas estériles en la célula hipógina de la rama carpogonial, detalles de la célula de fusión en el carposporofito y el patrón de desarrollo de los espermatangios son desconocidos para los ejemplares brasileños. Aunque el trabajo de Joly (1965) para $S$. furcellata no mencionó el patrón de disposición de los utrículos en vista superficial, de acuerdo con la figura 250 (Joly 1965, pág. 115), se verifica que los utrículos son de tamaño uniforme (córtex del grupo de $S$. moniliformis).

De acuerdo con Huisman (1985), Scinaia furcellata ocurre en el Atlántico oriental (del sur de Noruega hasta Marruecos, Islas Canarias y Mediterráneo). Huisman (1985) estudió el material de S. furcellata procedente de Irlanda y Francia y demostró que en vista superficial, los utrículos más grandes son circundados por células más pequeñas (pigmentadas o incoloras) o sea, con apariencia de roseta. Por el patrón de distribución de los utrículos, se concluyó que la identificación de Joly (1965) para el material brasileño, como $S$. furcellata, fue incorrecta.

Los caracteres taxonómicos analizados permiten que el material estudiado pueda ser identificado como Scinaia halliae (localidad tipo no especificada en Setchell 1914). Ganesan (1974) trabajó con esta especie con ejemplares provenientes de Venezuela; las descripciones y figuras presentadas concuerdan con los datos del material brasileño. 
Se examinaron ejemplares de Scinaia brasiliensis descritos por Joly et al. (1965), con base en material recolectado en Alagoas (SPF 2016 - isotipo) y se verificó que se trata de la misma especie de Espírito Santo (Guimarães 2006) y Bahia, o sea, S. halliae. Cuando Joly et al. (1965) propusieron la especie, establecieron que $S$. brasiliensis (como Pseudogloiophloea brasiliensis) difería de $S$. halliae por ser dioica y por presentar cistocarpos más grandes. A través del estudio del material tipo de $S$. brasiliensis, se verificó que los ejemplares de Alagoas son monoicos, lo contrario de lo establecido por Joly et al. (1965), y la mayor dimensión de los cistocarpos fue de $210 \mu \mathrm{m}$ de longitud y $200 \mu \mathrm{m}$ de diámetro. Por lo tanto, las dos características establecidas por Joly et al. (1965) para proponer la nueva especie no han sido confirmadas y no se encontró ninguna característica que separase S. brasiliensis de S. halliae. Joly et al. (1965) mencionaron e iconografiaron la rama carpogonial con 4-5 células, lo que también se constituye en una interpretación errónea, pues el género Scinaia presenta rama carpogonial con 3 células (Huisman 1986, Kajimura 1988).

De esta forma, el estudio detallado presentado en este trabajo confirma que Scinaia brasiliensis Joly \& Cordeiro-Marino es sinónimo taxonómico de $S$. halliae (Setchell) Huisman, conforme lo propuesto por Guimarães (2006).

\section{Agradecimientos}

Al PICDT - CAPES por la concesión de la beca de PósGraduación, a las Empresas CETREL y ENSER el Paso por la cesión del material proveniente del infralitoral.

\section{Literatura citada}

Betancourt-Fernandez L \& A Herrera-Moreno. 2001. Algas marinas bentónicas (Rhodophyta, Phaeophyta y Chlorophyta) conocidas para la Hispaniola. Moscosoa 12: 105-134.

Díaz-Pulido G \& M Díaz-Ruíz. 2003. Diversity of the benthic marine algae of the Colombian Atlantic. Biota Colombiana 4: 203-246.

Dixon PS \& LM Irvine. 1977. Seaweeds of the British Isles. Part 1 Introduction, Nemaliales Gigartinales, 252 pp. British Museum (Natural History), London.

Ganesan EK. 1974. Studies on the marine algal flora of Venezuela. V. Pseudogloiophlea halliae. Journal of Phycology 10: 415-418.

Ganesan EK. 1990. A catalog of benthic marine algae and seagrasses of Venezuela, 237 pp. Fondo Editorial Conicit Caracas.
Guimarães SMPB. 2006. A revised checklist of benthic marine Rhodophyta from the State of Espírito Santo, Brazil. Boletim do Instituto de Botânica 17: 145-194.

Guiry MD. 2009. AlgaeBase. World eletronic publication. [en línea] <http://www.algaebase.com>

Hanisack MD \& SM Blair. 1988. The deep water macroalgal community of the East Florida Continental Shelf (USA). Helgolänger Meeresuntersuchungen 42: 133-163.

Huisman JM. 1985. The Scinaia assemblage (Galaxauraceae, Rhodophyta): a re-appraisal. Phycologia 24: 403-418.

Huisman JM. 1986. The red algal genus Scinaia (Galaxauraceae, Nemaliales) from Australia. Phycologia 25 (3): 271-296.

Huisman JM, JT Harper \& GW Saunders. 2004. Phylogenetic study of the Nemaliales (Rhodophyta) based on large-subunit ribosomal DNA sequence supports segregation of the Scinaiaceae fam, nov. and the resurrection of Dichotomaria Lamarck. Phycological Research 52: 224234.

Joly AB, M Cordeiro-Marino, Y Ugadim, N YamaguishiTomita \& FC Pinheiro. 1965. New marine algae from Brazil. Arquivos da Estação de Biologia Marinha da Universidade Federal do Ceará 5(2): 79-92.

Joly AB, Y Ugadim, EC Oliveira-Filho, FC Pinheiro \& MM Ferreira. 1966. Additions to the marine flora of Brazil. VII. Arquivos da Estação de Biologia Marinha da Universidade Federal do Ceará 6: 51-57.

Kajimura M. 1988. Three new deep-water species of Scinaia (Galaxauraceae, Rhodophyta) from the Sea of Japan. Botanica Marina 31: 175-185.

Lee Y \& YD Ko. 2006. The red algal genus Scinaia (Galaxauraceae, Nemaliales) on Jeju Island, Korea. Algae 21(3): 267-281.

León-Cisneros K, R Riosmena-Rodriguez, AI Neto \& G Hernández-Carmona. 2004. The red algal genus Scinaia (Nemaliales; Rhodophyta) on the Gulf of California, Mexico: a taxonomic account. Phycologia 48(3): 186-210.

Ortega MM, JL Godinez \& GG Solórzano. 2001. Catálogo de las algas bénticas de las costas mexicanas, del Golfo de México y Mar de Caribe. Instituto de Biologia, Universidad Nacional Autónoma de México. Cuadernos 34: 1-594.

Robledo D, Y Freire-Pelegrín \& I Sánchez-Rodriguez. 2003. Marine benthic algae from the Campeche Banks, México, pp. 257-262. Proceedings of the XVII International Seaweed Symposium. Oxford University Press, Oxford.

Schnetter R \& G Bula-Meyer. 1978. Rodoficeas nuevas para la costa Atlántica de Colombia II. Anales del Instituto de Investigaciones Marinas de Punta Betín 10: 95-99. 
Setchell WA. 1914. The Scinaia assemblage. University of California Publications in Botany 6: 79-152.

Taylor WR. 1943. Marine algae from Haiti collected by H.H. Bartlett in 1941. Papers of the Michigan Academy of Science 28: 143-63.
Taylor WR. 1960. Marine algae of the Eastern Tropical and Subtropical coasts of the Americas, 870 pp. Michigan Press, Ann Harbor.

Recibido el 02 de junio de 2009 y aceptado el 21 de diciembre de 2009 